\title{
ON THE SIERPIŃSKI-ERDÖS AND THE OXTOBY-ULAM THEOREMS FOR SOME NEW SIGMA-IDEALS OF SETS ${ }^{1,2}$
}

\author{
C. G. MENDEZ
}

\begin{abstract}
Let $\Phi(\Psi)$ denote the family of subsets of the unit square defined to be of first category (Lebesgue measure zero) in almost every vertical line in the sense of measure (category). THEOREM 1. There is a homeomorphism of the unit square onto itself mapping a given set in $\Phi(\Psi)$ onto a set of Lebesgue measure zero. THEOREM 2. There is a set belonging to both $\Phi$ and $\Psi$ that cannot be mapped onto a set of first category by a homeomorphism of the unit square onto itself.

Let $C$ denote the Cantor set, regarded as the product of a sequence of 2-element groups, and let $\Lambda$ denote one of the $\sigma$-ideals of subsets of $C$ studied by Schmidt and Mycielski. TheOREM 3. Assuming the continuum hypothesis, the Sierpinski-Erdös theorem holds for $\Lambda$ and the class of subsets of $C$ of Haar measure zero (or of first category). THEOREM 4. The Oxtoby-Ulam theorem holds for the image of $\Lambda$ under the Cantor mapping of $C$ onto the unit interval.
\end{abstract}

1. On the Sierpiński-Erdös theorem. This section is inspired by the work of Schmidt [8] and Mycielski [4] on $\sigma$-ideals of sets on $C$ (the Cantor set), or for that matter on $I$ (the unit interval).

In correspondence with Mycielski's definition we have: Given a set $S \subset C$ and a set $K$ of natural numbers we define a positional game $\Gamma(S, K)$ with perfect information between two players I and II. The players choose consecutive terms of a sequence $\left(x_{0}, x_{1}, x_{2}, \ldots\right) \in C$ (where $C$ is hereby regarded as the topological space $\left(\prod_{i=1}^{\infty} X_{i}, \prod_{i=1}^{\infty} T_{i}\right), X_{i}=\{0,1\}$, and $T_{i}$ is the discrete topology on $X_{i}$ ), the choice $x_{i}$ is made by player I if $i \notin K$, and by player II if $i \in K$. The player choosing $x_{i}$ knows $S, K$, and $x_{0}, x_{1}, \ldots, x_{i-1}$.

Player I wins if $\left(x_{0}, x_{1}, x_{2}, \ldots\right) \in S$, and player II wins in the other case.

Let $W_{\mathrm{II}}(K)$ be the class of sets $S \subset C$ for which player II has a winning strategy in the game $\Gamma(S, K)$. Let $M=\left\{K_{s_{1}, s_{2}, \ldots, s_{n}}: s_{i}=0,1 ; 1 \leqslant i \leqslant n\right.$, $n \in J\}$ be a system of sets of natural numbers such that $K_{s_{1}, s_{2}, \ldots, s_{n}, s_{n+1}}$ is

Presented to the Society, April 16, 1978; received by the editors October 2, 1976 and, in revised form, December 14, 1977.

AMS (MOS) subject classifications (1970). Primary 54A05; Secondary 28A65, 90D05.

Key words and phrases. Measure zero, first category, ideals of sets, infinite games, winning strategies, homeomorphisms, Sierpinski-Erdös theorem, Oxtoby-Ulam theorem.

'Some of the results in this article are contained in the author's Ph.D. dissertation submitted to the University of Colorado, Boulder, in December of 1974 and written under the direction of Professor S. M. Ulam.

${ }^{2}$ The author wishes to acknowledge a debt of gratitude to the Society's referee who reviewed this manuscript for her/his invaluable help and suggestions. 
contained in $K_{s_{1}, s_{2}, \ldots, s_{n}}$ and $K_{s_{1}, s_{2}, \ldots, s_{n-1}, 0} \cap K_{s_{1}, s_{2}, \ldots, s_{n-1}, 1}=0$ for $n \in J$. Let $\Lambda=\bigcap W_{\mathrm{II}}\left(K_{s_{1}, s_{2}}, \ldots, s_{n}\right)$ where the intersection runs over all the sets $K_{s_{1}, s_{2}, \ldots, s_{n}}$ of the system $M$. We shall assume that all sets $K_{s_{1}, s_{2}, \ldots, s_{n}}$ of the system $M$ are nonempty and hence infinite.

Now we would like to show the following properties for $\Lambda$.

1.1 Theorem. (i) $\Lambda$ is $\sigma$-ideal.

(ii) The union of $\Lambda$ is $C$.

(iii) The complement of each member of $\Lambda$ contains a set of power $c$ belonging to $\Lambda$.

(iv) $C$ may be represented as the union of two complementary Borel sets one in $\Lambda$ and the other one of first category and measure zero where the measure in $C$ is the Haar measure; or equivalently, the measure induced by the Lebesgue measure in the unit interval I and the Cantor mapping of $C$ onto $I$.

(v) There is a subclass $\Upsilon$ of power $\leqslant c$ of $\Lambda$ such that each member of $\Lambda$ is contained in some member of the subclass $\Upsilon$.

Proof. (i) Shown in Mycielski's paper as Theorem 3.

(ii) Obvious from Theorem 4 in Mycielski's paper.

(iii) Given $S \in \Lambda$ we define the set $A$ as follows:

$A=\left\{x: \quad x=\left(x_{0}, x_{1}, x_{2}, \ldots\right), x_{j_{k}^{0}}=f_{j_{k}^{0}}\left(x_{0}, x_{1}, x_{2}, \ldots, x_{j_{k}^{0}-1}\right)\right.$ where

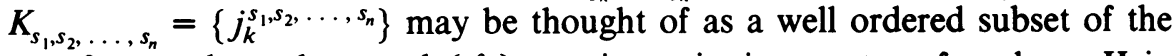
set of natural numbers and $\left\{f_{j_{k}^{0}}\right\}_{j_{k}^{0} \in K_{0}}$ is a winning strategy for player II in game $\Gamma\left(S, K_{0}\right)$, and $x_{j_{n}, s_{2} \ldots s_{n}}=0$ for all $n \in J$ and $\left.\left\{1, s_{2}, \ldots, s_{n}\right\}\right\}$.

We would like to show:

(a) $A \subset S^{c}$,

(b) $A \in \Lambda$,

(c) $A$ is of power $c$.

Proof of (a). $x=\left(x_{0}, x_{1}, \ldots\right) \in A \Rightarrow x_{j_{k}^{0}}=f_{j_{k}^{0}}\left(x_{0}, x_{1}, \ldots, x_{j_{k}^{0}-1}\right)$ where $\left\{f_{j_{k}^{0}}\right\}_{j_{k}^{0} \in K_{0}}$ is a winning strategy for player II in game $\Gamma\left(S, K_{0}\right)$. (This is possible for there is such a winning strategy for $S \in \Lambda$.) Hence $x \in S^{c}$.

Proof of (b). To show that for any given $K_{s_{1}, s_{2}, \ldots, s_{n}} \in M$, there is a winning strategy $\left\{g_{j}\right\}_{j \in K_{s_{1}, s_{2} \ldots s_{n}}}$ for player II in game $\Gamma\left(A, K_{s_{1}, s_{2}, \ldots, s_{n}}\right)$. If

$$
K_{s_{1}, s_{2}, \ldots, s_{n}} \subset K_{0}\left(K_{s_{1}, s_{2}, \ldots, s_{n}}=K_{0, s_{2}, \ldots, s_{n}}\right) \text {, }
$$

then $j_{1}^{0, s_{2}}, \ldots, s_{n}=j_{i}^{0}$ for some $i$; and we define $g_{j}$ such that

$$
\begin{aligned}
& x_{j_{i}^{0}}=x_{j_{1}^{0}, s_{2} \ldots s_{n}}=g_{j_{1}^{0, s_{2}} \ldots, s_{n}}\left(x_{0}, \ldots, x_{j_{1}^{0}, s_{2} \ldots, s_{n-1}}\right) \\
& =g_{j i}\left(x_{0}, \ldots, x_{j_{i}^{0}-1}\right) \neq f_{j_{i}^{0}}\left(x_{0}, \ldots, x_{j_{i}^{0}-1}\right)
\end{aligned}
$$

for that $i$, hence $x=\left(x_{0}, \ldots, x_{j_{i}-1}, x_{j_{i}^{0}}, \ldots\right) \notin A$.

On the other hand, if $K_{s_{1}, s_{2}, \ldots, s_{n}} \in K_{1}\left(K_{s_{1}, s_{2}, \ldots, s_{n}}=K_{1, s_{2}, \ldots, s_{n}}\right)$, then define

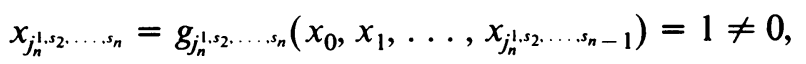

hence $x=\left(x_{0}, x_{1}, \ldots, x_{j_{n}^{1, s_{2}} \ldots, s_{n}}, \ldots\right) \notin A$. 
Proof of (c). Define

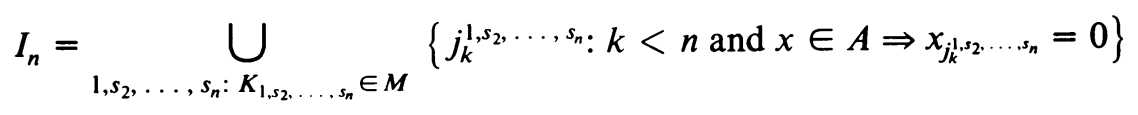

and

$$
J_{n}=\bigcup_{1, s_{2}, \ldots, s_{n}: K_{1, s_{2} \ldots, s_{n}} \in M}\left\{j_{k}^{\left.1, s_{2}, \ldots, s_{n}: k<n\right\}}\right.
$$

then the power of $I_{n} \leqslant 2^{0}+2^{1}+2^{2}+\cdots+2^{n-2}$, and the power of $J_{n}=$ $(n-1) 2^{n-1}$.

That is, the power of $I_{n}<2^{n-1}$, and the power of $J_{n}>2^{n}$ for $n \geqslant 4$. Hence there is a sequence of integers $\left\{j_{n}\right\}_{4}^{\infty}$ such that $j_{n} \neq j_{i}, i<n, j_{n} \in J_{n}$, and $j_{n} \notin I_{n}$, since $2^{n-1}+(n-4)<2^{n}, n \geqslant 4$.

Therefore $x_{j_{n}}$ may be chosen to be 0 or 1 for any $x$ such that

$$
x_{j_{k}^{0}}=\left(f_{j_{k}^{0}}\left(x_{0}, x_{1}, \ldots, x_{j_{k}^{0}-1}\right)\right) \text { and } x_{j_{n}^{1, s_{2}} \ldots, s_{n}}=0 \text {. }
$$

That is, the power of $A$ is $c$.

(iv) As in Mycielski's paper we define $S_{M}$ to be the set of all $x=\left(x_{0}\right.$, $\left.x_{1}, \ldots\right) \in C$ such that for every $K_{s_{1}, s_{2}, \ldots, s_{n}}$ of $M$ there is some $i \in$ $K_{s_{1}, s_{2}}, \ldots, s_{n}: x_{i}=0$. Then the proof of (iv) is obvious.

(v) Obvious from Theorem 10 in Mycielski's paper. Q.E.D.

1.2 Theorem. Assuming the continuum hypothesis, the Sierpinski-Erdös theorem is satisfied for $\Lambda$ and the class of sets of measure zero (first category) in $C$ as defined above.

Proof. 1.1 Theorem and [5, p. 76, Theorem 19.6]. Q.E.D.

\section{On the Oxtoby-Ulam theorem.}

A. On mixed sigma-ideals. In 1938 J. C. Oxtoby and S. M. Ulam [6] used to so-called category method to show the existence of a homeomorphism of the unit interval onto itself mapping a given set of first category onto a set of measure zero [5, p. 50]. In this section we shall show the corresponding counterparts of this statement for $\Phi(\Psi)$ (the family of subsets of the unit square defined to be of first category (Lebesgue measure zero) in almost every vertical line in the sense of measure (category) [3, pp. 124-128] employing a similar technique based on an argument provided by the same authors in proving a generalization of the same theorem in 1941 [7, p. 912].

2.1 THEOREM. There is a homeomorphism of the unit square onto itself mapping a given set in $\Phi(\Psi)$ onto a set of measure zero.

Proof. If $K \in \Phi$ then there is a measure zero set $S$ in $I$ such that $K_{x}$ is of first category for every $x$ in $I-S$. Moreover, $K$ may be represented as the union of two complementary sets: $A=\{(x, y) \in K: x \in I-S\}$ and $B=$ $\{(x, y) \in K: x \in S\}$, such that $K \subset A \cup(S \times I), I^{2}-(S \times I)$ (where $I^{2}=I \times I$ ) is dense in $I^{2}$ (for $S \times I$ is a set of measure zero) and $A_{x}$ is of first category for every $x$. 
Now, let $I^{2}(x)=\left\{(x, y) \in I^{2}\right.$, for some fixed $\left.x \in I\right\}$, then for every $x \in I-S$ there is a sequence $\left\{P_{n}^{x}\right\}_{1}^{\infty}$ of perfect subsets of $I^{2}(x)$ contained in $I^{2}-(A \cup(S \times I))$ whose union is dense in $I^{2}(x)[7$, p. 912].

Now, let us consider the countable double sequence $P_{n}^{d}, n \in J, d \in D$, where $D$ is any countable dense subset of $I-S$ (this is possible, for $I-S$ is a separable metric space as a subset space of $I$ ) of perfect subsets of $I^{2}(d)$, and hence of $I^{2}$ (for $I^{2}(d)$ is a closed subset of $\left.I^{2}\right)$; contained in $I^{2}-(A \cup$ $(S \times I)$ ), and hence in $I^{2}-K$; whose union is dense in $D \times I$ and therefore in $I^{2}$ (since $D \times I$. is dense in $I^{2}$ ). Therefore we have exhibited a sequence of perfect subsets of $I^{2}$ contained in the complement of $K$ whose union is dense in $I^{2}$ and established an equivalent condition to this theorem [7, p. 912]. A similar argument for $\Psi$ shall be omitted. Q.E.D.

2.2 THEOREM. There is a set belonging to both $\Phi$ and $\Psi$ that cannot be mapped onto a set of first category by a homeomorphism of the unit square onto itself.

Proof. $I^{2}=(A \times A) \cup(B \times B) \cup(A \times B) \cup(B \times A)$, where $\{A, B\}$ denotes a partition of $I$ into an $F_{\sigma}$ set of first category and a $G_{\delta}$ set of measure zero [5, p. 5]. And hence, $(A \times A) \cup(B \times B) \in \Phi(\Psi)$ and not of first category in $I^{2}$ (for $(A \times B) \cup(B \times A)$ the complement of $(A \times A) \cup$ $(B \times B)$ is of first category [5, pp. 54, 57]). Therefore, no homeomorphic image of $(A \times A) \cup(B \times B)$ is of first category in $I^{2}$; and hence, the corresponding counterpart of the Oxtoby-Ulam theorem fails. Q.E.D.

B. On the ideals of Schmidt and Mycielski. Throughout the rest of this section, $B$ shall define the binary sequence of subsets of $[0,1]$ with the right-hand end points (except 1) excluded; that is, $B_{n}$ is the set of points in $[0,1]$ whose $n$th binary digit is 1 , taking the terminating expansion in the case of dyadic rationals, except for 1 . More formally, $B_{n}=\left\{x:\left(x_{1}, x_{2}, \ldots, x_{n-1}\right.\right.$, $\left.1, x_{n+1}, \ldots\right) \in[0,1]: x_{i}=0,1$ and there is no $m \in J: x_{i}=1$, for $i>m$, except for $x=1$.

Moreover, $c_{B}(I)$ shall identify the image of $I$ in $C$ under the Marezewski function of the binary sequence $B$, given by:

$$
c_{B}(x)=\sum_{n=1}^{\infty} \frac{2 \chi_{B_{n}}(x)}{3^{n}}, \quad x \in I,
$$

and $\chi_{B_{n}}$ the characteristic function of $B_{n}$, for all $B_{n} \in B[9$, p. 210].

Note that $c_{B}^{-1}$ is the restriction of the Cantor function to $c_{B}(I)$ which turns out to be the Cantor set with the left-hand endpoints of the contiguous intervals removed. Hence, $c_{B}^{-1}$ is a one-to-one and continuous function from $c_{B}(I)$ onto $I$.

Since the Oxtoby-Ulam theorem takes place in $I$, we shall redefine our $\sigma$-ideal $\Lambda$ as the collection $\zeta=\left\{c_{B}^{-1}\left(S \cap c_{B}(I)\right): S \in \Lambda\right\}$ of subsets of $I$.

\subsection{THEOREM. $\zeta$ is a $\sigma$-ideal of subsets of $I$.}


Proof. Given a sequence $S_{n}$ in $\Lambda$,

$$
\begin{aligned}
\bigcup_{n} c_{B}^{-1}\left(S_{n} \cap c_{B}(I)\right) & =c_{B}^{-1}\left(\bigcup_{n}\left(S_{n} \cap c_{B}(I)\right)\right) \\
& =c_{B}^{-1}\left(\left(\bigcup_{n} S_{n}\right) \cap c_{B}(I)\right) \in \zeta,
\end{aligned}
$$

for $\cup S_{n}$ is in $\Lambda$ ( $\Lambda$ being a $\sigma$-ideal).

Moreover, if $T \subset c_{B}^{-1}\left(S \cap c_{B}(I)\right)$, for a given set $S$ in $\Lambda$, then

$$
c_{B}(T) \subset c_{B}\left(c_{B}^{-1}\left(S \cap c_{B}(I)\right)\right)=S \cap c_{B}(I) \subset S,
$$

for $c_{B}^{-1}$ is one-to-one. Hence, $c_{B}(T)$ is in $\Lambda$, for $S$ is in $\Lambda$ ( $\Lambda$ being a $\sigma$-ideal). But

$$
T=c_{B}^{-1}\left(c_{B}(T)\right)=c_{B}^{-1}\left(c_{B}(T) \cap c_{B}(I)\right)
$$

for $c_{B}^{-1}$ is onto, and hence $T$ is in $\zeta$. Q.E.D.

2.4 THEOREM. Given $S$ in $C$ such that $S$ is in $\Lambda$, then there is a sequence of perfect subsets of $C$ contained in the complement of $S$ whose union is dense in $C$.

Proof. Given $S$ in $\Lambda$, and $K_{s_{1}, s_{2}, \ldots, s_{n}}$ in $M$ as introduced above we define $P_{s_{1}, s_{2}, \ldots, s_{n}}=\left\{z=\left(z_{0}, z_{1}, \ldots\right): z_{j}=0\right.$ or 1 , and $z_{j}=f_{j}\left(z_{0}, z_{1}, \ldots, z_{j-1}\right)$ for $j$ in $K_{s_{1}, s_{2}, \ldots, s_{n}}$, where $\left\{f_{j}\right\}_{j \in K_{s_{1}, s_{2}, \ldots . s_{n}}}$ is a winning strategy for player II in game $\left.\Gamma\left(S, K_{s_{1}, s_{2}, \ldots, s_{n}}\right)\right\}$.

We would like to show $\left\{P_{s_{1}, s_{2}, \ldots, s_{n}}\right\}_{S_{1,1}, \ldots, s_{n} \in M}$ is a sequence of perfect subsets of $C$ contained in the complement of $S$ whose union is dense in $C$.

(a) $P_{s_{1}, s_{2}, \ldots, s_{n}}$ is closed.

PROOF OF (a). If $x$ is in the complement of $P_{s_{1}, s_{2}, \ldots, s_{n}}$, then $x_{j} \neq f_{j}\left(x_{0}\right.$, $\left.x_{1}, \ldots, x_{j-1}\right)$ for some $j$ in $K_{s_{1}, s_{2}, \ldots, s_{n}}$. Moreover, if $N_{x}=\left\{y \in I: y_{k}=x_{k}\right.$, for all $k \leqslant j\}$, then $N_{x}$ defines a neighborhood of $x$ contained in the complement of $\boldsymbol{P}_{s_{1}, s_{2}, \ldots, s_{n}}$. Hence, the complement of $\boldsymbol{P}_{s_{1}, s_{2}, \ldots, s_{n}}$ is open.

(b) $P_{s_{1}, s_{2}, \ldots, s_{n}}$ is dense in itself.

PRoOF OF (b). Suppose $x$ is in $P_{s_{1}, s_{2}, \ldots, s_{n}}$, i.e., $x_{j}=f_{j}\left(x_{0}, x_{1}, \ldots, x_{j-1}\right)$ for all $j$ in $K_{s_{1}, s_{2}}, \ldots, s_{n}$, and hence for $j$ in $K_{s_{1}, s_{2}, \ldots, s_{n}}$ such that $j \leqslant m$, for any $m$.

Now, given $\varepsilon>0$, choose $y$ to agree with $x$ for finitely and sufficiently many entries (say, $y_{j}=x_{j}$ for $j \leqslant m$ ) such that $d(x, y)<\varepsilon$. On the other hand, for $j>m$ choose $y_{j}$ such that $y_{j}=f_{j}\left(y_{0}, y_{1}, \ldots, y_{j-1}\right)$ for $j$ in $K_{s_{1}, s_{2}, \ldots, s_{n}}, y_{k} \neq x_{k}$ for $k$ equal to the first integer $>m: k \notin K_{s_{1}, s_{2}, \ldots, s_{n}}$, and $y_{k}=x_{k}$ for all other $k \notin K_{s_{1}, s_{2}, \ldots, s_{n}}$. This is possible for $K_{s_{1}, s_{2}, \ldots, s_{n}}$ cannot include every integer from some point on, otherwise $K_{s_{1}, s_{2}, \ldots, s_{n-1}, 0 \text { or } 1}$ were to finite. A contradiction.

Hence $y=\left(y_{0}, y_{1}, \ldots, y_{j}, \ldots\right): y_{j}=f_{j}\left(y_{0}, y_{1}, \ldots, y_{j-1}\right)$ for all $j \in$ $K_{s_{1}, s_{2}, \ldots, s_{n}}$, i.e., $y \in P_{s_{1}, s_{2}, \ldots, s_{n}}$ and $x \neq y$.

Therefore, if $x$ is in $P_{s_{1}, s_{2}, \ldots, s_{n}}$, then $x$ is a limit point of $P_{s_{1}, s_{2}, \ldots, s_{n}}$. 
(c)

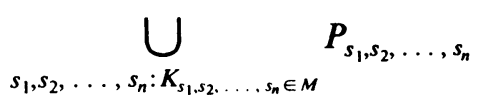

is dense in $C$.

Proof OF (c). Let $x \in C$. Given $\varepsilon>0$, choose $y$ to agree with $x$ for finitely and sufficiently many entries (say, $y_{j}=x_{j}$ for $j \leqslant m$ ) such that $d(x, y)<\varepsilon$.

Now consider $s_{1}, s_{2}, \ldots, s_{n}: j \in K_{s_{1}, s_{2}, \ldots, s_{n}} \Rightarrow j>m$. This is possible, since $K_{s_{1}, s_{2}, \ldots, s_{n+1}} \subset K_{s_{1}, s_{2}, \ldots, s_{n}}$, and $K_{s_{1}, s_{2}, \ldots, s_{n-1}, 0} \cap K_{s_{1}, s_{2}, \ldots, s_{n-1}, 1}=0$.

Now define $y: y_{j}=f_{j}\left(y_{0}, y_{1}, \ldots, y_{j-1}\right)$, if $j \in K_{s_{1}, s_{2}}, \ldots, s_{n}$ (and note that $j>m$ ), and $y_{j}=x_{j}$, for all $j \notin K_{s_{1}, s_{2}, \ldots, s_{n}}$.

Then

$$
y \in P_{s_{1}, s_{2}, \ldots, s_{n}} \Rightarrow y \in \cup P_{s_{1}, s_{2}, \ldots, s_{n}} .
$$

Therefore, $x \in I$, hence $x \in \overline{\cup P_{s_{1}, s_{2}, \ldots, s_{n}}}$.

(d) $P_{s_{1}, s_{2}}, \ldots, s_{n}$ is contained in the complement of $S$.

Proof OF (d). If $z$ is in $P_{s_{1}, s_{2}, \ldots, s_{n}}$, then $z_{j}=f_{j}\left(z_{0}, z_{1}, \ldots, z_{j-1}\right), j \in$ $K_{s_{1}, s_{2}, \ldots, s_{n}}$ and hence $z=\left(z_{0}, z_{1}, \ldots\right)$ is in the complement of $S$. Q.E.D.

Whether an analogue of the Oxtoby-Ulam theory holds for $\Lambda$, that is, whether for each $S \in \Lambda$ there exists an automorphism $h$ of $C$ such that $h(S)$ has Haar measure zero. remains an open question. However, such a theorem holds for $\zeta$.

2.5 THEOREM. For each $S \in \zeta$ there is a homeomorphism $h$ of I onto I such that $h(S)$ has Lebesgue measure zero.

Proof. $c_{B}(S)$ belongs to $\Lambda$, hence by 2.4 Theorem there is a sequence $\left\{P_{i}\right\}$ of perfect sets contained in $C-c_{B}(S)$ whose union is dense in $C$. Because $C-c_{B}(I)$ is countable, each of the sets $P_{i} \cap c_{B}(I)$ contains a sequence $\left\{P_{i, j}\right\}, j \in J$, of compact and perfect sets whose union is dense in $P_{i}$. Hence $\left\{P_{i, j}\right\}$ is a countable family of compact and perfect subsets of $c_{B}(I)-c_{B}(S)$ whose union is dense in $C$. Because $c_{B}^{-1}$ is one to one and continuous on $c_{B}(I)$, it follows that $\left\{c_{B}^{-1}\left(P_{i, j}\right)\right\}$ is a countable family of compact and perfect subsets of $I-S$ whose union is dense in $I$. The conclusion then follows from [7, p. 912]. Q.E.D.

\section{REFERENCES}

1. P. R. Halmos, Measure theory, Van Nostrand, Princeton, N.J., 1950.

2. K. Kuratowski, Topology, Vol. I, Academic Press, New York, 1966.

3. C. G. Mendez, On sigma-ideals of sets, Proc. Amer. Math. Soc. 60 (1976), 124-128.

4. J. Mycielski, Some new ideals of sets on the real line, Colloq. Math. 20 (1969), 71-76.

5. J. C. Oxtoby, Measure and category, Springer-Verlag, New York, 1971.

6. J. C. Oxtoby and S. M. Ulam, On the equivalence of any set of first category to a set of measure zero, Fund. Math. 31 (1938), 201-206. 
7. Measure-preserving homeomorphisms and metrical transitivity, Ann. of Math. (2) 43 (1941), 874-920.

8. W. Schmidt, On badly approximable numbers and certain games, Trans. Amer. Math. Soc. 123 (1966), 178-199.

9. E. Szpilrajn, The characteristic function of a sequence of sets and some of its applications, Fund. Math. 31 (1938), 207-225.

Department of Mathematical Sciences, Metropoltan State College, Denver, COLORADO 80204 